\title{
Gene expression profiling in mouse embryonic stem cells reveals glycogen synthase kinase-3-dependent targets of phosphatidylinositol 3-kinase and Wnt/ $\beta$-catenin signaling pathways
}

\author{
Colleen M. Bartman ${ }^{1}$, Jennifer Egelston ${ }^{1}$, Sravya Kattula ${ }^{1}$, Leigh C. Zeidner ${ }^{2}$, Anthony D'Ippolito ${ }^{2 \dagger}$, \\ Bradley W. Doble ${ }^{3}$ and Christopher J. Phiel ${ }^{1 *}$ \\ 1 Department of Integrative Biology, University of Colorado Denver, Denver, CO, USA \\ ${ }^{2}$ Center for Human and Molecular Genetics, Research Institute at Nationwide Children's Hospital, Columbus, OH, USA \\ ${ }^{3}$ Stem Cell and Cancer Research Institute, Department of Biochemistry and Biomedical Sciences, McMaster University, Hamilton, ON, Canada
}

Edited by:

Marc Lee Goalstone, University of Colorado Denver, USA

\section{Reviewed by:}

Kazunori Sango, Tokyo Metropolitan Institute of Medical Science, Japan Zhong Q Wang, Pennington

Biomedical Research Center, USA

\section{*Correspondence:}

Christopher J. Phiel, Department of Integrative Biology, University of

Colorado Denver, Campus Box 171,

P.O. Box 173364, Denver, CO

80217-3364, USA

e-mail: christopher.phie/@

ucdenver.edu

${ }^{\dagger}$ Present address:

Anthony D'Ippolito, Duke Institute for Genome Sciences \& Policy, Duke University, Durham, NC, USA
Glycogen synthase kinase-3 (Gsk-3) activity is an important regulator of numerous signal transduction pathways. Gsk-3 activity is the sum of two largely redundant proteins, Gsk$3 \alpha$ and Gsk-3 $\beta$, and in general, Gsk-3 is a negative regulator of cellular signaling. Genetic

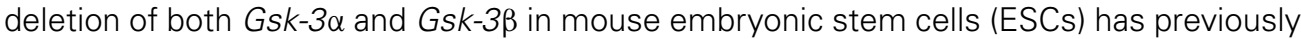
been shown to lead to the constitutive activation of the $\mathrm{Wnt} / \beta$-catenin signaling pathway. However, in addition to Wnt signaling, all Gsk-3-regulated pathways, such as insulin sig-

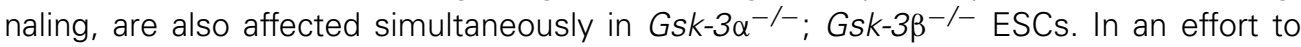
better understand how specific signaling pathways contribute to the global pattern of gene

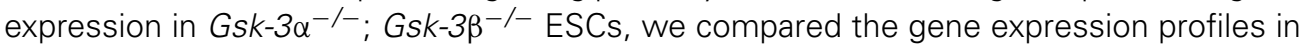

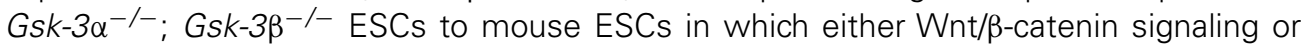
phosphatidylinositol 3-kinase (PI3K)-dependent insulin signaling are constitutively active. Our results show that Wnt signaling has a greater effect on up-regulated genes in the Gsk-

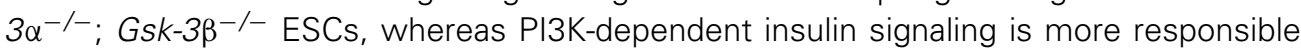
for the down-regulation of genes in the same cells. These data show the importance of Gsk-3 activity on gene expression in mouse ESCs, and that these effects are due to the combined effects of multiple signaling pathways.

Keywords: embryonic stem cells, glycogen synthase kinase-3, Wnt, phosphatidylinositol 3-kinase, microarray, quantitative PCR, signal transduction, gene expression

\section{INTRODUCTION}

Glycogen synthase kinase-3 (Gsk-3) is an intracellular serine/threonine kinase activity that was originally identified as the rate-limiting step in the glycogen synthesis pathway (1). When the genes encoding Gsk-3 were cloned, Gsk-3 activity was revealed

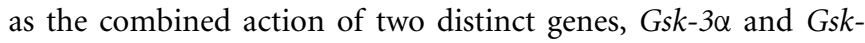
$3 \beta$ (2). Gsk-3 isoforms show a high degree of similarity at the amino acid level, including $98 \%$ similarity within the catalytic domain. Despite divergence at the amino- and carboxyl-termini of each isoform, it appears that the unstructured carboxyl-termini are essential for Gsk-3 activity (3). Rare among kinases, Gsk-3 is active at a basal state, while pathway activation from upstream signaling cascades results in the inhibition of Gsk-3 activity (4-6).

There are two primary mechanisms responsible for Gsk-3 inhibition - one that regulates the phosphorylation of Gsk-3 $\alpha$ and

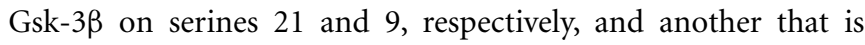
independent of this phosphorylation event. Several signaling pathways, including protein kinase A (PKA), Hedgehog, transforming growth factor- $\beta$ (TGF- $\beta$ ), nuclear factor of activated T-cells (NF$\mathrm{AT}$ ), and phosphatidylinositol 3-kinase (PI3K)-dependent insulin signaling, all affect Gsk-3 activity via phosphorylation of the amino-terminal serines (4-6). Insulin binding to its receptor triggers the activation of PI3K, which phosphorylates and activates Akt, which in turn phosphorylates and inhibits Gsk-3 activity (7). We have previously shown that stable expression of a constitutively active form of the p110 $\alpha$ catalytic subunit of PI3K (termed p110*) (8) in WT mouse ESCs can effectively lead to the phosphorylation of Gsk-3 (9), and expression of p $110^{*}$ has been shown to activate insulin signaling in mouse ESCs (10).

Gsk-3 activity also plays a central role in Wnt signaling as it is part of a cytosolic protein complex termed the $\beta$-catenin destruction complex (11). The role of Gsk-3 is to directly phosphorylate $\beta$-catenin $(12,13)$, which targets $\beta$-catenin for ubiquitin-mediated degradation (14), keeping the Wnt pathway inactive. Activation of the Wnt signaling pathway inhibits Gsk-3 activity, but this effect is not mediated through amino-terminal serine phosphorylation. Instead, upon Wnt ligand binding to a Frizzled/Lrp receptor complex, the destruction complex is neutralized via the translocation of Gsk-3 to the cell membrane, where it phosphorylates Lrp (15, 16). Gsk-3 is then subsequently endocytosed into multivesicular bodies, further insulating it from $\beta$-catenin (17). Once $\beta$-catenin protein levels accumulate to high levels in the cytoplasm, it is 
subsequently translocated to the nucleus where it can directly complex with lymphocyte enhancer factor (Lef)/T-cell factor (Tcf) proteins to activate Wnt target genes $(18,19)$. Gsk-3 $\alpha$ and Gsk$3 \beta$ are redundant with respect to their roles in regulating Wnt signaling; the genetic deletion of either $G s k-3 \alpha$ or $G s k-3 \beta$ alone is insufficient to activate Wnt signaling (20-25). Only upon the deletion of three of the four total alleles for Gsk-3 $\alpha$ and Gsk-3 $\beta$ is Wnt pathway activation achieved (23). Double knockout of both

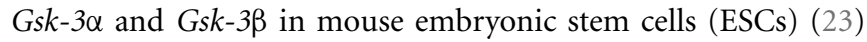
or mouse neural progenitors (26) results in the constitutive activation of the Wnt pathway. Importantly, while Gsk-3 regulates both insulin and Wnt signaling, these pathways do not activate one another; Wnt signaling does not activate insulin signaling and insulin signaling does not activate Wnt signaling (27-29).

Lithium is a direct inhibitor of Gsk-3 activity $(30,31)$ and is used therapeutically for the treatment of bipolar disorder (32). The inhibition of Gsk-3 activity has also been shown to be a potential means to reduce the pathology associated with Alzheimer's disease (33-38). Because the dysregulation of Gsk-3 activity is believed to be an important contributor to many diseases, including Alzheimer's disease, cancer, mental illness, and diabetes, the acquisition of more fundamental knowledge about the effects of reduced Gsk-3 activity could be important in the context of these diseases.

In this paper, we describe the analysis of genome-wide gene

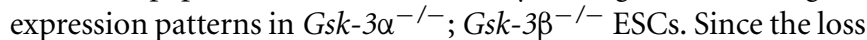
of Gsk-3 activity results in the activation of numerous signaling pathways simultaneously, we further dissected the effects of losing Gsk-3 activity by examining gene expression patterns in ESCs with either constitutively active PI3K-mediated insulin signaling or constitutively active Wnt signaling.

\section{MATERIALS AND METHODS}

\section{MOUSE EMBRYONIC STEM CELL CULTURE}

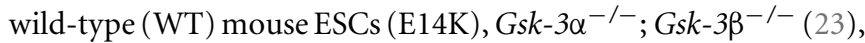
p110* (9), and $\beta$-catenin S33A (39) stable cell lines, along with respective control WT ESCs stably transfected with puromycinresistant plasmids, were grown on $0.1 \%$ gelatin-coated plates in high glucose DMEM (Life Technologies) supplemented with 15\% fetal bovine serum (Hyclone), 1\% non-essential amino acids, 1\% sodium pyruvate, $1 \%$ L-glutamine, $1 \%$ penicillin/streptomycin (Life Technologies), $55 \mu \mathrm{M}$ 2-mercaptomethanol, and $1000 \mathrm{U} / \mathrm{ml}$ ESGRO (Millipore). Media was replenished every other day.

\section{RNA ISOLATION, cDNA SYNTHESIS, AND QUANTITATIVE PCR}

RNA was isolated from $5 \times 10^{5}$ to $1 \times 10^{6}$ ESCs using the MirVana Total RNA Isolation Kit (Applied Biosystems) according to the manufacturer's instructions. Two micrograms of RNA were then used for cDNA synthesis using the high capacity cDNA reverse transcription kit (Applied Biosystems) following the manufacturer's protocol. Quantitative RT-PCR was performed on an Applied Biosystems StepOne machine using TaqMan master mix and one of the following TaqMan assays (Applied Biosystems): Axin2 (Mm00443610_m1), Brachyury (Mm00436877_m1), Bhmt1 (Mm04210521_g1), Bhmt2 (Mm00517726_m1), Cdx2 (Mm01212280_m1), Ido2 (Mm00524206_m1), Anxa8 (Mm00507926_m1), Aqp8 (Mm0043 1846_m1), Gata6(Mm00802636_m1),Wnt6 (Mm00437351_m1),
Acta2 (Mm01546133_m1), or Foxq1 (Mm01157333_s1). Three biological replicates and three technical replicates were used for each cell type. All threshold cycle $(\mathrm{Ct})$ values were normalized to a mouse Gapdh endogenous control (Mm99999915_g1) (Applied Biosystems), and relative quantification was calculated from the median Ct value (40). $P$-values were calculated using Data Assist software (Applied Biosystems).

\section{MICROARRAY ANALYSIS}

RNA was isolated using the MirVana Total RNA Isolation Kit (Applied Biosystems) according to the manufacturer's instructions. The concentration of RNA was measured in a Nanodrop ND-1000 UV-Vis spectrophotometer (Nanodrop) and an Agilent 2100 Bioanalyzer Lab-On-A-Chip Agilent 6000 Series II chip (Agilent) was used to determine the integrity of the samples. RNA from each cell line was hybridized onto a SurePrint G3 Mouse GE $8 \times 60 \mathrm{~K}$ Microarray, $8 \times 60 \mathrm{~K}$, AMADID 028005 (Agilent). Hybridization was performed overnight at $45^{\circ} \mathrm{C}$. We performed arrays for each cell line using RNA that was isolated in biological triplicate $(n=3)$. For Gsk-3 DKO ESCs, we used $n=4$ biological replicates. SurePrint arrays were scanned with an Agilent G2505C Microarray Scanner (Agilent). The information about each probe on the arrays was extracted from the image data using Agilent Feature Extraction 10.9 (FE) and .txt files were generated. The raw intensity values from these files in imported into the mathematical software package "R," which was used for all data input, diagnostic plots, normalization, and quality checking steps of the analysis process using scripts developed by Dr. Peter White, Director of the Biomedical Genomics Core at Nationwide Children's Hospital specifically for this analysis. These scripts call on several Bioconductor packages, an open-source and open-development software project to provide tools for the analysis and comprehension of genomic data (41). The algorithm used for normalization of gene expression was designed for use with Agilent One-Color Analysis. The median green $(\mathrm{Cy} 3)$ intensities are normalized between the arrays using the Quantile Normalization package in " $R$ " (42). Quantile normalization is a non-linear probe-level normalization that results in the same empirical distribution of intensities for each array. This is a significantly more robust approach than simply normalizing to the median value of each array. The genes that were altered by twofold either way and had a false discovery rate (FDR) of $<10 \%$ were sorted and used for further interpretation of the microarray data.

\section{STATISTICAL ANALYSIS OF MICROARRAY DATA}

Statistical analyses were performed using two well-validated and commonly used approaches - significance analysis of microarrays (SAM) and adjusted $p$-value. SAM is a powerful tool for analyzing microarray gene expression data useful for identifying differentially expressed genes between two conditions (43). SAM calculates a test statistic for relative difference in gene 5 expression based on permutation analysis of expression data and calculates a FDR using the $q$-value method presented in Ref. (44). In outline, SAM identifies statistically significant genes by carrying out gene specific $t$-tests and computes a statistic for each gene, which measures the strength of the relationship between gene expression and a response variable. This analysis uses non-parametric statistics, 
since the data may not follow a normal distribution. The response variable describes and groups the data based on experimental conditions. In this method, repeated permutations of the data are used to determine if the expression of any gene is significant related to the response. The use of permutation-based analysis accounts for correlations in genes and avoids parametric assumptions about the distribution of individual genes. For this experiment, SAM analysis was implemented in R using the Bioconductor Siggenes package. In a one-color experimental design, a two-class unpaired analysis is typically performed for each experimental comparison, whereas in a two-color approach a one-class analysis is used. Typically, an FDR cutoff in the range of $10-20 \%$ is chosen to maximize sensitivity without significantly impacting accuracy. For the current study, a 10\% FDR was used to generate the list of significantly differentially expressed genes.

\section{Adjusted p-value}

The moderated $t$-statistic $(t)$ is computer for each probe and for each contrast in the experimental design. This has the same interpretation as an ordinary $t$-statistic except that the standard errors have been moderated across genes, i.e., shrunk toward a common value. This has the effect of borrowing information from the ensemble of genes to aid with inference about each individual gene. The $p$-value is obtained from the distribution of the moderated $t$-statistic. Finally these $p$-values are adjusted for multiple testing using the Benjamini and Hochberg's (45) step-up method for controlling the FDR. This is the most popular method for $p$-value adjustment. If all genes with $p$-value below a threshold, say 0.05 , are selected as differentially expressed, then the expected proportion of false discoveries in the selected group is controlled to be less than the threshold value, in this case $5 \%$. For the current study, the adjusted $p$-values were calculated using the Bioconductor limma package.

\section{GENE ENRICHMENT ANALYSIS}

After filtering gene lists to remove duplicates, the HGNC symbols for genes increased or decreased by twofold or more were entered into ToppFun, within the ToppGene Suite (toppgene.cchmc.org) (46). ToppGene then performs functional enrichments, looking for sets of co-regulated genes. Calculations are performed using FDR for correction, and a $p$-value cutoff of $<0.05$.

\section{RESULTS}

We had previously analyzed the genome-wide expression in Gsk$3 \alpha^{-1-}$; Gsk-3$\beta^{-1-}$ ESCs (hereafter referred to as Gsk-3 double knockout; Gsk-3 DKO) using Affymetrix microarrays, and found hundreds of genes whose expression was significantly increased or decreased compared to WT ESCs (9). Here, we repeated this microarray analysis using the Agilent platform. For all of the microarray gene expression analyses, we used a twofold or greater change in gene expression as our threshold for significance. We also isolated RNA and performed the hybridization to the microarrays at the same time to reduce experiment-to-experiment variability. Consistent with our previous results using the Affymetrix platform, we found 1313 genes up-regulated twofold or more in Gsk-3 DKO ESCs, while 2178 genes were down-regulated twofold or more (a complete list of genes can be found in Datasheet 1 in Supplementary Material). One of the important signaling pathways regulated by Gsk-3 is the Wnt pathway (47). We therefore expected our microarray data from Gsk-3 DKO ESCs to reveal many of the same Wnt target genes that have been demonstrated experimentally in a variety of model systems [Wnt Homepage, www.stanford.edu/group/ nusselab/cgi-bin/wnt/target_genes]. A few well-established direct targets of Wnt signaling, such as Brachyury (T) and Axin2, were found to be increased substantially in Gsk-3 DKO ESCs (162.5and 8.6-fold, respectively) (Table 1). We were surprised, however, to find that Brachyury and Axin2 were the exception of the $65 \mathrm{Wnt}$ putative target genes; we identified in our microarray data from Gsk-3 DKO ESCs that only seven additional genes ( $S p 5, C d x 1$, Stra6, Lef1, Cyclin D1, PTTG, and Fgf18) had their expression increased more than twofold (Table 1). In fact, $13 \mathrm{Wnt}$ target genes showed decreased expression of more than twofold (Table 1).

The relative paucity of up-regulated Wnt target genes was surprising, especially, since it has been shown that a reporter construct containing multimerized Lef/Tcf binding sites is strongly activated in Gsk-3 DKO ESCs [(23); unpublished observation]. Therefore, we wanted to compare the gene expression data from Gsk-3 DKO ESCs with another mouse embryonic stem cell line that has constitutively active Wnt signaling. WT ESCs stably expressing a form of $\beta$-catenin in which serine 33 has been mutated to an alanine (S33A) that prevents the phosphorylation by Gsk-3 on this residue as well as subsequent ubiquitination, resulting in the constitutive activation of Wnt signaling. These cells have previously been shown to potently activate the expression of several Wnt target genes (39). The $\beta$-catenin S33A cells and their control cells were included in our microarray experiment to investigate how similar the patterns of gene expression were compared to Gsk-3 DKO ESCs.

Microarray data showed that 1468 genes were up-regulated twofold or more in S33A cells compared to control cells, while 1412 genes were down-regulated twofold or more. As expected, we confirmed the high levels of Brachyury, Axin2, and $C d x 1$ that were also observed in the Gsk-3 DKO ESCs; however, only three additional genes ( $S p 5, C d x 4$, and VEGF) showed a twofold or greater increase in gene expression in the S33A ESCs (Table 1). And similar to the data from Gsk-3 DKO ESCs, 12 Wnt target genes are downregulated twofold or more in S33A cells (Table 1). Taken together, these data show that some Wnt target genes, such as Brachyury and Axin2, are indeed transcribed at high levels in two different cell lines in which Wnt signaling is constitutively active - Gsk-3 DKO and $\beta$-catenin S33A. Our data also show that many Wnt target genes are not activated in these cell lines as was predicted.

Initially, we had expected that many of the genes whose expression was significantly increased in Gsk-3 DKO ESCs would be due to activation of the Wnt pathway. Since we did not observe the increased expression of Wnt target genes, we asked whether the activation of another Gsk-3-dependent signaling pathway could explain the data. Because the genetic deletion of Gsk-3 isoforms likely leads to the activation of signaling pathways other than the Wnt pathway, we speculated that the combined effect of multiple pathway activation likely leads to new patterns of gene expression. Another important signal transduction pathway that is regulated by Gsk-3 activity is the insulin signaling pathway. To investigate the contribution of insulin signaling to the effects on gene 
Table 1 | Comparison of known Wnt target genes to their expression levels in Gsk-3 DKO and S33A ESCs as determined by microarray.

\begin{tabular}{|c|c|c|}
\hline Gene & DKO & S33A \\
\hline Brachyury & 162.5 & 41.7 \\
\hline SP5 & 75.8 & 15.1 \\
\hline $\mathrm{Cdx1}$ & 16.5 & 82.5 \\
\hline Axin-2 & 8.6 & 4.6 \\
\hline Stra6 & 4.8 & 1.3 \\
\hline LEF1 & 4 & -7 \\
\hline Cyclin D & 3.1 & -3.5 \\
\hline Pituitary tumor transforming gene (PTTG) & 2.2 & 1.8 \\
\hline FGF18 & 2.1 & -2.9 \\
\hline BMP4 & 1.8 & 1.2 \\
\hline PPAR $\delta$ & 1.6 & 1.1 \\
\hline Endothelin-1 & 1.5 & 1.4 \\
\hline Telomerase & 1.5 & 1.4 \\
\hline LGR5/GPR49 & 1.5 & -1.2 \\
\hline Jagged & 1.4 & 1 \\
\hline sFRP-2 & 1.4 & -1.5 \\
\hline CD44 & 1.3 & 1.4 \\
\hline c-myc binding protein & 1.3 & -1.9 \\
\hline Msl1 & 1.3 & 1.2 \\
\hline Nitric oxide synthase 2 & 1.3 & 1.4 \\
\hline$N k \times 2.2$ & 1.3 & 1.2 \\
\hline$c-m y c$ & 1.2 & -3.3 \\
\hline Met & 1.2 & -1.2 \\
\hline Claudin-1 & 1.1 & 1 \\
\hline ld2 & 1.1 & 1.2 \\
\hline FoxN1 & 1.1 & 1.1 \\
\hline Gbx2 & 1.1 & -2.2 \\
\hline MMP-7 & 1 & 1 \\
\hline Osteoprotegerin & 1 & 1 \\
\hline Wnt3a & 1 & 1.2 \\
\hline Neurogenin 1 & 1 & 1.1 \\
\hline Tiam1 & -1.1 & -1.2 \\
\hline FGF20 & -1.1 & -1.4 \\
\hline $\mathrm{Cdx} 4$ & -1.1 & 2.3 \\
\hline Sox17 & -1.2 & -1.6 \\
\hline Oct-4 & -1.2 & -1.3 \\
\hline Runx2 & -1.3 & 1 \\
\hline Sox2 & -1.3 & 1.4 \\
\hline Frizzled 7 & -1.3 & -2.1 \\
\hline Gastrin & -1.4 & 1.3 \\
\hline Sox9 & -1.4 & -1.1 \\
\hline CCN1/Cyr61 & -1.4 & -1.2 \\
\hline Follistatin & -1.4 & 1.2 \\
\hline NeuroD1 & -1.5 & -5.6 \\
\hline c-jun & -1.6 & -2.2 \\
\hline $\mathrm{Nr}-\mathrm{CAM}$ & -1.6 & -1.5 \\
\hline SALL4 & -1.6 & -2.3 \\
\hline Nanog & -1.6 & 1.4 \\
\hline FGF9 & -1.7 & 1 \\
\hline Irx3 and Six3 & -1.8 & -1.4 \\
\hline Cacna1 $\gamma$ & -1.8 & -1.8 \\
\hline
\end{tabular}

(Continued)
Table 1 | Continued

\begin{tabular}{llc}
\hline Gene & DKO & S33A \\
\hline MMP2, MMP9 & -1.9 & 1.6 \\
LBH & -2 & 1.4 \\
Pitx2 & -2.1 & -1.5 \\
ITF-2 & -2.3 & -1.9 \\
Twist & -2.4 & -2 \\
Periostin & -2.4 & 1 \\
VEGF & -2.5 & 2.4 \\
Snail & -2.8 & -1.5 \\
Tcf-1 (Hnf1a) & -3 & -1.1 \\
n-myc & -3.4 & -3.4 \\
Gremlin & -3.5 & -3.5 \\
EGF receptor & -3.8 & -1.5 \\
Delta-like 1 & -9.8 & -4.3 \\
WISP & -10 & -1.1 \\
\hline
\end{tabular}

The list of genes was found at The Wnt Homepage website (www.stanford.edu/ group/nusselab/cgi-bin/wnt/target_genes). Genes whose expression increased more than twofold are shown in red, while genes whose expression decreased more than twofold are shown in green.

expression seen in the Gsk-3 DKO ESCs, we analyzed genome-wide microarray gene expression data from WT ESCs stably expressing a constitutively active form of the p110 subunit of PI3K (p110*) $(9,10)$. Eight hundred sixty-four genes were up-regulated twofold or more in p $110^{\star}$ ESCs compared to control cells, while 1660 genes were down-regulated twofold or more.

After compiling the list of genes whose expression was significantly changed in Gsk-3 DKO, p110*, and S33A ESCs, we were curious as to the similarities and disparities in the patterns of gene expression in these cell lines. To perform this evaluation, we removed all duplicate probes from our lists of genes from the microarray experiment, and we then sorted based on changes seen in each list. The Gsk-3 DKO and p110* ESCs had 1313 and 864 genes, respectively, that were up-regulated twofold or more. Of these, 206 genes were up-regulated in both cell types (Figure 1A). Similarly, 1468 genes were up-regulated by at least twofold in S33A cells, and 336 of these genes overlapped with the 1313 genes upregulated in the Gsk-3 DKO ESCs (Figure 1A). As we expected, there was relatively little overlap in the genes up-regulated in both p110* and S33A ESCs; only 119 genes were in common between these gene sets (Figure 1A). This comparison of up-regulated genes shows a greater degree of overlap between Gsk-3 DKO and S33A ESCs than with Gsk-3 DKO and p110* ESCs.

Interestingly, the similarities between gene sets were even greater when examining the genes whose expression was downregulated twofold or more. Two thousand one hundred seventyeight genes showed decreased expression in Gsk-3 DKO ESCs, compared with 1660 genes in p110* ESCs and 1412 genes in S33A ESCs. Furthermore, we found that 987 genes were in common between $\mathrm{p} 110^{*}$ and Gsk-3 DKO ESCs, while only 516 genes were shared between S33A and Gsk-3 DKO ESCs (Figure 1B). This pattern is striking in that it is the opposite of what was seen with the up-regulated genes, with a greater overlap between Gsk-3 DKO and $\mathrm{p} 110^{*}$ ESCs. In addition, the overlap between $\mathrm{p} 110^{*}$ and 


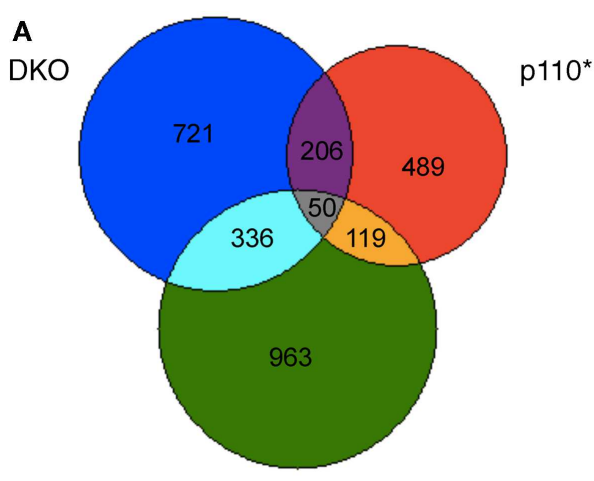

S33A
B

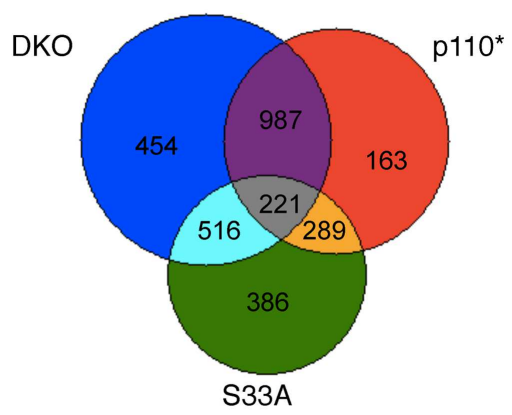

FIGURE 1 | Venn diagram representing the overlap in genes whose expression is changed at least twofold in Gsk-3 DKO, p110*, and $\beta$-catenin S33A ESCs. (A) Representation of genes whose expression was increased in Gsk-3 DKO (blue; 1313 genes total), p110* (red; 864 genes total), and S33A ESCs (green; 1468 genes total). Genes whose expression is increased in multiple genes sets are denoted by the numbers at the interface of the gene sets. (B) Representation of genes whose expression was decreased in Gsk-3 DKO (blue; 2178 genes total), p110* (red; 1660 genes total), and S33A ESCs (green; 1412 genes total). Genes whose expression is decreased in multiple genes sets are denoted by the numbers at the interface of the gene sets.
S33A ESCs was also more pronounced, with the same 289 genes down-regulated at least twofold in both cell lines (Figure 1B). These data suggest that the loss of Gsk-3 in ESCs results in the upregulation of mostly distinct genes in either p110* or S33A ESCs, but that those genes that are down-regulated in the absence of Gsk-3 share a greater overlap in cells with activated PI3K signaling and activated Wnt signaling.

Since different sets of genes were either increased or decreased in expression in p110* and S33A ESCs, we bioinformatically performed enrichment analyses to see if functionally related genes were co-regulated in a signaling pathway-specific fashion (Tables 2 and 3). Genes that were up-regulated in Gsk-3 DKO ESCs were involved in gene ontology (GO): molecular function of protein domain (GO:0019904) and also the GO: biological process for cilium organization (GO:0044782). In addition, genes encoding for CD molecules, tumor necrosis factor receptor superfamily, and intraflagellar transport homologs were up-regulated. Genes that were increased in expression in p110* ESCs showed an enrichment for the GO: molecular function of piRNA binding (GO:0034584) and an showed an increase in expression in genes encoding for zinc fingers, $\mathrm{C} 2 \mathrm{H} 2$, and BTB domain-containing (ZBTB). In addition, both Gsk-3 DKO and p110* ESCs showed increased expression of genes involved in urothelium and lower urinary tract development. For genes up-regulated in S33A ESCs, the top GO: molecular function clusters were genes involved in organic acid binding (GO:0043177) and protein homodimerization activity (GO:0042803). Also, a significant number of genes that contain homeobox domains were up-regulated in the S33A ESCs, as well as a high number of genes encoding for CD molecules.

We found it notable that the enrichments among downregulated genes were more robust than among the up-regulated genes (Table 4). For genes decreased twofold or more in Gsk-3 DKO ESCs, the greatest enrichment for GO: molecular function was in genes encoding for sequence-specific DNA binding, i.e., transcription factors (GO:0043565). In addition, a large number of genes involved in the development of the cardiovascular system (GO:0072358) were down-regulated in Gsk-3 DKO ESCs. Also decreased in expression were genes involved in extracellular matrix organization and elastic fiber formation. For downregulated genes in $110^{\star}$ ESCs, the most enriched GO: molecular function was for genes involved in receptor binding (GO:0005102), while the highest enrichment for GO: biological processes were for organ morphogenesis (GO: 0009887), extracellular matrix organization (GO:0030198), and cardiovascular system development (GO:0072358). Furthermore, many genes involved in extracellular matrix organization were decreased in p110* ESCs. Genes down-regulated in S33A ESCs were enriched for genes encoding sequence-specific DNA binding (GO:0043565), as well as enrichment for genes involved in neurogenesis (GO:0022008) and cardiovascular system development (GO:0072358). The results from these enrichment analyses showed several similarities and differences with respect to the genes that have increased or decreased expression in Gsk-3 DKO, p110*, and S33A ESCs, and provide a framework for beginning to better understand the complex interrelationships between PI3K-mediated signaling and Wnt signaling, as well as providing important insights into the patterns of gene expression seen in Gsk-3 DKO ESCs.

While the data obtained from microarray experiments can provide an excellent overall view of differential patterns of gene expression, it is nonetheless very important to use an independent technique to experimentally validate the changes in gene expression that were observed via microarray. Therefore, we selected a handful of genes whose expression patterns we of interest, and we performed quantitative reverse-transcriptase PCR (qPCR) using TaqMan probes on RNA isolated from Gsk-3 DKO, p110*, S33A ESCs, and their respective control cell lines. As an initial quality control measure, we performed qPCR to validate the expression of the known Wnt target genes, Axin2 and Brachyury. As expected, we saw substantial increases in gene expression in both Gsk-3 DKO and S33A ESCs for Axin2 (up 7-fold in Gsk-3 DKO and 3.7-fold 
Table 2 | Summary of enrichment analysis of genes up-regulated in Gsk-3 DKO, p110*, and S33A ESCs.

\begin{tabular}{lll}
\hline Name & ID & $p$-Value
\end{tabular}

\section{GO: molecular function}

Protein domain specific binding GO:0019904 $1.01 \times 10^{-5} \quad 55 / 633$

\section{GO: biological process}

Cilium organization

$$
\text { GO:0044782 } 6.06 \times 10^{-6} \quad 19 / 123
$$

\section{Gene family}

CD molecules

Tumor necrosis factor receptor

superfamily

Intraflagellar transport homologs

$\begin{array}{ccc}\text { CD } & 5.14 \times 10^{-8} & 21 / 276 \\ \text { TNFRSF } & 5.77 \times 10^{-5} & 4 / 12 \\ & & \\ \text { IFT } & 1.52 \times 10^{-4} & 4 / 15\end{array}$

\section{Coexpression Atlas}

Mendel_RNAseq_e17.5_urothelium

Developing lower urinary

tract_e13.5

\section{P110* ESCs}

GO: molecular function

piRNA binding

$\begin{array}{ccc}\text { GO:0034584 } & 3.17 \times 10^{-5} & 3 / 3 \\ \text { ZBTB } & 5.33 \times 10^{-5} & 6 / 47\end{array}$

Gene family

Zinc fingers, $\mathrm{C} 2 \mathrm{H} 2$, and BTB

domain containing

$2.74 \times 10^{-16} \quad 101 / 989$

$1.04 \times 10^{-13} \quad 82 / 802$

\section{Coexpression Atlas}

Mendel_RNAseq_e17.5_urothelium

Developing lower urinary

tract_e13.5

\section{S33A ESCs}

\section{GO: molecular function}

Organic acid binding

Protein homodimerization activity

\section{Gene family}

CD molecules

$\begin{array}{lll}\text { GO:0043177 } & 4.93 \times 10^{-5} & 26 / 230 \\ \text { GO:0042803 } & 5.15 \times 10^{-5} & 56 / 674\end{array}$

$1.58 \times 10^{-10} \quad 65 / 989$ $1.30 \times 10^{-6} \quad 30 / 802$
Bonferroni correction method.

All p-Value cutoffs - 0.05 .

in S33A ESCs) and Brachyury (up 622-fold in Gsk-3 DKO and 38-fold in S33A ESCs), while both genes has reduced expression in p110* ESCs (0.4- and 0.7-fold changes, respectively) (Figure 2). These results are consistent with Axin2 and Brachyury being Wnt target genes.

We then proceeded to validating the expression of additional genes whose expression levels were shown to be substantially increased or reduced in the microarray experiments. We selected four genes, Bhmt1 (Betaine-homocysteine methyltransferase), Bhmt2 (Betaine-homocysteine methyltransferase 2), Cdx2 (Caudal-type homeobox 2), and Ido2 (Indoleamine 2,3-dioxygenase 2), whose expression, by microarray, was shown to be up-regulated
Table 3 | Summary of enrichment analysis of genes down-regulated in Gsk-3 DKO, p110*, and S33A ESCs.

\begin{tabular}{|c|c|c|c|}
\hline Name & ID & $p$-Value & Hit count \\
\hline
\end{tabular}

\section{GO: molecular function}

Sequence-specific DNA binding GO:0043565 $8.59 \times 10^{-13} \quad 109 / 726$ Sequence-specific DNA binding GO:0003700 $7.28 \times 10^{-12} \quad 141 / 1067$ transcription factor activity

\section{GO: biological process}

Cardiovascular system development

GO:00722358 $2.15 \times 10^{-21} \quad 148 / 871$

\section{Pathway}

Extracellular matrix organization

Elastic fiber formation

$$
1.40 \times 10^{-9} \quad 50 / 264
$$$$
1.51 \times 10^{-7} \quad 15 / 41
$$

\section{P110* ESCs}

\section{GO: molecular function}

Receptor binding

GO:0005102 $1.51 \times 10^{-11} \quad 133 / 1341$

\section{GO: biological process}

Organ morphogenesis

Extracellular matrix organization

Cardiovascular system

GO:0009887 $2.09 \times 10^{-34} \quad 147 / 876$

GO:0030198 $1.59 \times 10^{-22} \quad 70 / 330$

development

GO:0072358 $2.34 \times 10^{-22}$

$124 / 871$

\section{Pathway}

Extracellular matrix organization

$6.17 \times 10^{-16}$

$54 / 264$

\section{S33A ESCs}

GO: molecular function

Sequence-specific DNA binding

Sequence-specific DNA binding

GO:0043565 $2.15 \times 10^{-10} \quad 81 / 726$

GO:0003700 $3.54 \times 10^{-10} \quad 105 / 1067$

transcription factor activity

\section{GO: biological process}

Neurogenesis

Extracellular matrix organization

GO:0022008 $3.88 \times 10^{-15} \quad 143 / 1360$ GO:0072358 $1.21 \times 10^{-14} \quad 104 / 871$

Bonferroni correction method.

All p-Value cutoffs - 0.05 .

in both Gsk-3 DKO and S33A ESCs, but not p110* ESCs. Validation by $\mathrm{qPCR}$ showed that the expression of Bhmt1 was up 14.1- and 70.1-fold in Gsk-3 DKO and S33A ESCs, respectively, compared to a modest 2.8 -fold increase in p110* ESCs (Figure 3). Similarly Bhmt2 expression was shown to be increased 13.1-fold in Gsk-3 DKO ESCs and 56.5-fold in S33A ESCs, while expression was only increased by 1.3 -fold in p110* ESCs (Figure 3). The expression of $C d x 2$ was increased 5.3- and 12.5-fold in Gsk-3 DKO ESCs and S33A ESCs, respectively, but remained unchanged in p110* ESCs (Figure 3). Finally, qPCR showed that Ido2 expression was increased 81.4-fold in Gsk-3 DKO ESCs and 20-fold in S33A ESCs, while showing a minimal 1.4 -fold increase in expression in $\mathrm{p} 110^{*}$ ESCs (Figure 3).

Next, we chose to perform similar qPCR validation on genes whose expression was changed in Gsk-3 DKO and p110* ESCs, but 
not in S33A ESCs. We selected six genes for analysis - Wnt6, Anxa8 (Annexin 8), Gata6 (GATA binding protein 6), Aqp8 (Aquaporin 8), Foxq1 (Forkhead box Q1), and Acta2 (actin, a2, smooth muscle, aorta). All of the genes that we assayed showed lower changes in gene expression compared to that seen by microarray. For example, microarray data showed that Wnt6 and Anxas were up-regulated

Table 4 | Summary of comparison between microarray and qPCR gene expression data in Gsk-3 DKO, S33A, and p110* ESCs for genes described in this work.

\begin{tabular}{|c|c|c|c|c|c|c|}
\hline & \multicolumn{2}{|l|}{ DKO } & \multicolumn{2}{|l|}{ S33A } & \multicolumn{2}{|c|}{ p110* } \\
\hline & Microarray & qPCR & Microarray & qPCR & Microarray & qPCR \\
\hline Axin2 & $6.7 \times$ & $7.0 \times$ & $3.6 \times$ & $3.7 \times$ & $1.7 \times$ & $-2.5 x$ \\
\hline Brachyury & $162.5 \times$ & $622 x$ & $41.7 \times$ & $38 x$ & $-1.5 x$ & $-1.4 \times$ \\
\hline Bhmt2 & $5.7 \times$ & $13.1 \times$ & $60.8 \times$ & $56.5 \times$ & $-2.6 \times$ & $1.3 \times$ \\
\hline $\mathrm{Cd} \times 2$ & 27.6 & $5.3 x$ & $33.3 x$ & $12.5 x$ & $1.2 \times$ & $1 \times$ \\
\hline Bhmt1 & $6.5 \times$ & $14.1 \times$ & $49.1 \times$ & $70.1 \times$ & $-5.5 x$ & $2.8 \times$ \\
\hline Ido2 & $9.4 \times$ & $81.4 \times$ & $3.4 \times$ & $20 x$ & $1.3 \times$ & $1.4 \times$ \\
\hline Wnt6 & $2.3 \times$ & $1.2 \times$ & $1.5 \times$ & $-1.4 \times$ & $10.1 \times$ & $5.7 \times$ \\
\hline An $\times a 8$ & $1.5 x$ & $1.7 \times$ & $1.5 \times$ & $1.4 \times$ & $6.8 x$ & $3.6 x$ \\
\hline Gata6 & $-1.4 \times$ & $-1.1 \times$ & -3.1 & $-2.5 x$ & $-31.8 x$ & $3.4 \times$ \\
\hline Aqp8 & $-1.5 x$ & $1.6 \times$ & -2.6 & $-3.3 x$ & $-48.9 x$ & $1.7 \times$ \\
\hline Fo $\times q 1$ & $-1.4 \times$ & $-1.1 \times$ & $-2.9 \times$ & $1.5 \times$ & $-31.5 x$ & $-1.7 \times$ \\
\hline Acta2 & $-400.3 x$ & $-5.0 \times$ & $-1.8 \times$ & $2.3 \times$ & $-165.6 \times$ & $-2.5 x$ \\
\hline
\end{tabular}

Decreases in gene expression are denoted by negative values. in p110* ESCs 10.1- and 6.8-fold, respectively; however, qPCR revealed that the expression of Wnt6 and Anxa8 was about half of that - 5.7- and 3.6-fold, respectively (Figure 4). Similarly, while Gata6, Aqp8, Foxq1, and Acta2 all showed at least 30-fold reductions in gene expression in p110* ESCs by microarray, the qPCR results revealed more subtle changes, ranging from a 3.4fold increase for Gata6 to a 2.5-fold reduction in Acta2 (Table 3). Importantly, the expression of these genes in S33A ESCs was quite different. For example, while Gata6 expression was up 3.4-fold in p110* ESCs, Gata6 expression was decreased 2.5-fold in S33A ESCs (Figure 5). Similarly, Acta2 expression was decreased 2.5fold in p110* ESCs, while Acta2 expression was increased 2.3-fold in S33A ESCs (Figure 5). These results are consistent with opposing effects of PI3K-mediated insulin signaling and Wnt signaling having distinct effects on gene expression.

\section{DISCUSSION}

The analysis of gene expression changes in Gsk-3 DKO ESCs revealed almost 3500 genes whose expression increased or decreased at least twofold, reinforcing the notion that Gsk-3 activity broadly regulates the expression of many genes. Our data, which examined global changes in gene expression in several clonal cell lines, illuminated numerous interesting and unexpected features of Gsk-3-dependent signal transduction.

We had initially expected to find many known Wnt target genes to be up-regulated in a Gsk-3 and $\beta$-catenin-dependent fashion and this was indeed true for Axin2 (48) and Brachyury. However, most of the other Wnt target genes did not show significant increases in expression, and in fact, almost twice as many

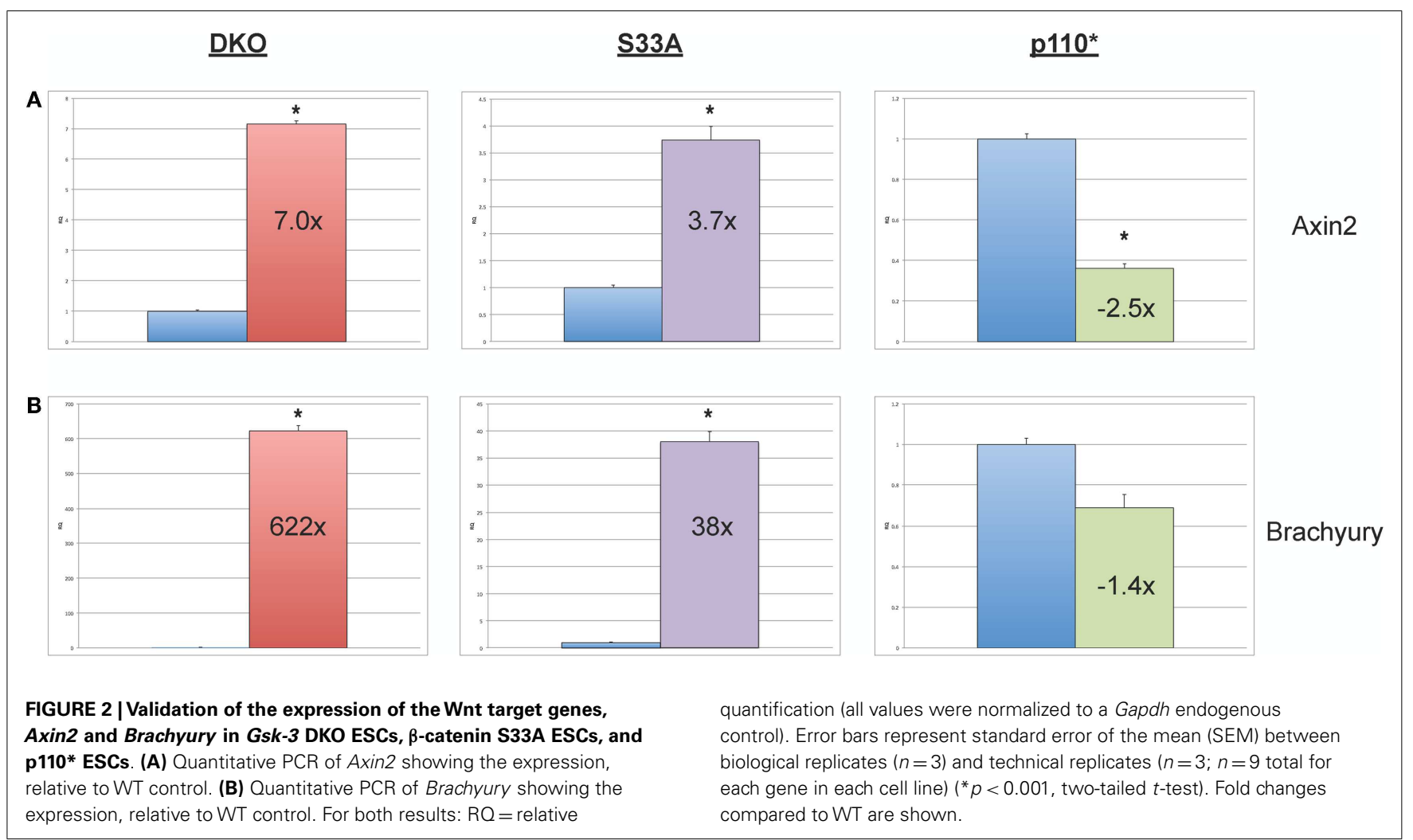




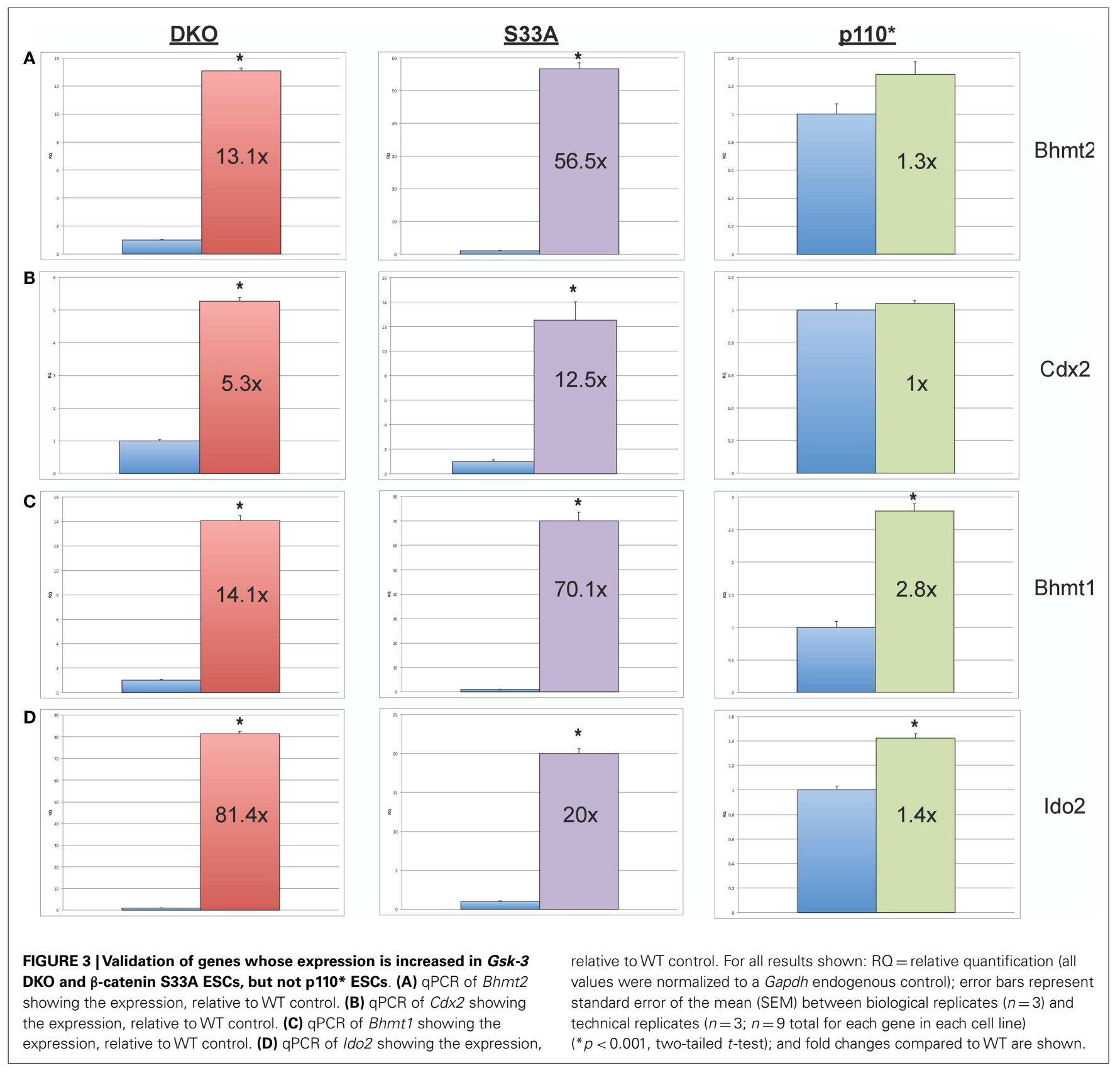

genes showed decreases in expression. It is possible that this simply reflects differences in the regulation of gene expression between cell types; alternatively, this data could provide a readout of affected genes due to the constitutive activation of Wnt

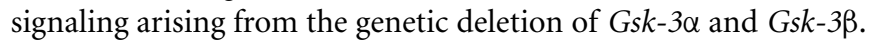

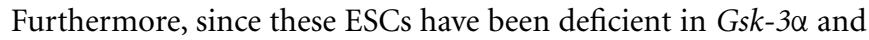
Gsk-3ß since their successful homologous recombination event, it is possible that downstream negative feedback effects serve to modify the expression of Wnt target genes, making the changes in gene expression not appear as dramatic as expected.

We do, however, identify several putative novel Wnt target genes, whose expression is increased in both Gsk-3 DKO and $\beta$-catenin S33A ESCs. Cdx2, Ido2, Bhmt1, and Bhmt2 were all confirmed by qPCR to conform to the microarray expression profiling. Interestingly, the transcription factor $C d x 2$, was recently shown to be a direct target of Wnt signaling in the mouse, independently confirming our observation (49). Furthermore, while Ido1 has been shown to be a Wnt target gene (50), Ido2 has not. In the mouse genome, the Ido1 and Ido2 genes lie next to each other on chromosome 8 (8qA2) and are transcribed in the same direction. Thus, one possibility is that $\beta$-catenin-mediated transcription could influence both genes from the Ido1 promoter. Alternatively, there may be additional Lef/Tcf binding sites in the Ido2 promoter. An in silico search $(51,52)$ for Lef/Tcf binding sites in the mouse Ido2 promoter identified eight putative binding sites within $2 \mathrm{~kb}$ of the transcription start site (TSS). Further detailed studies will 


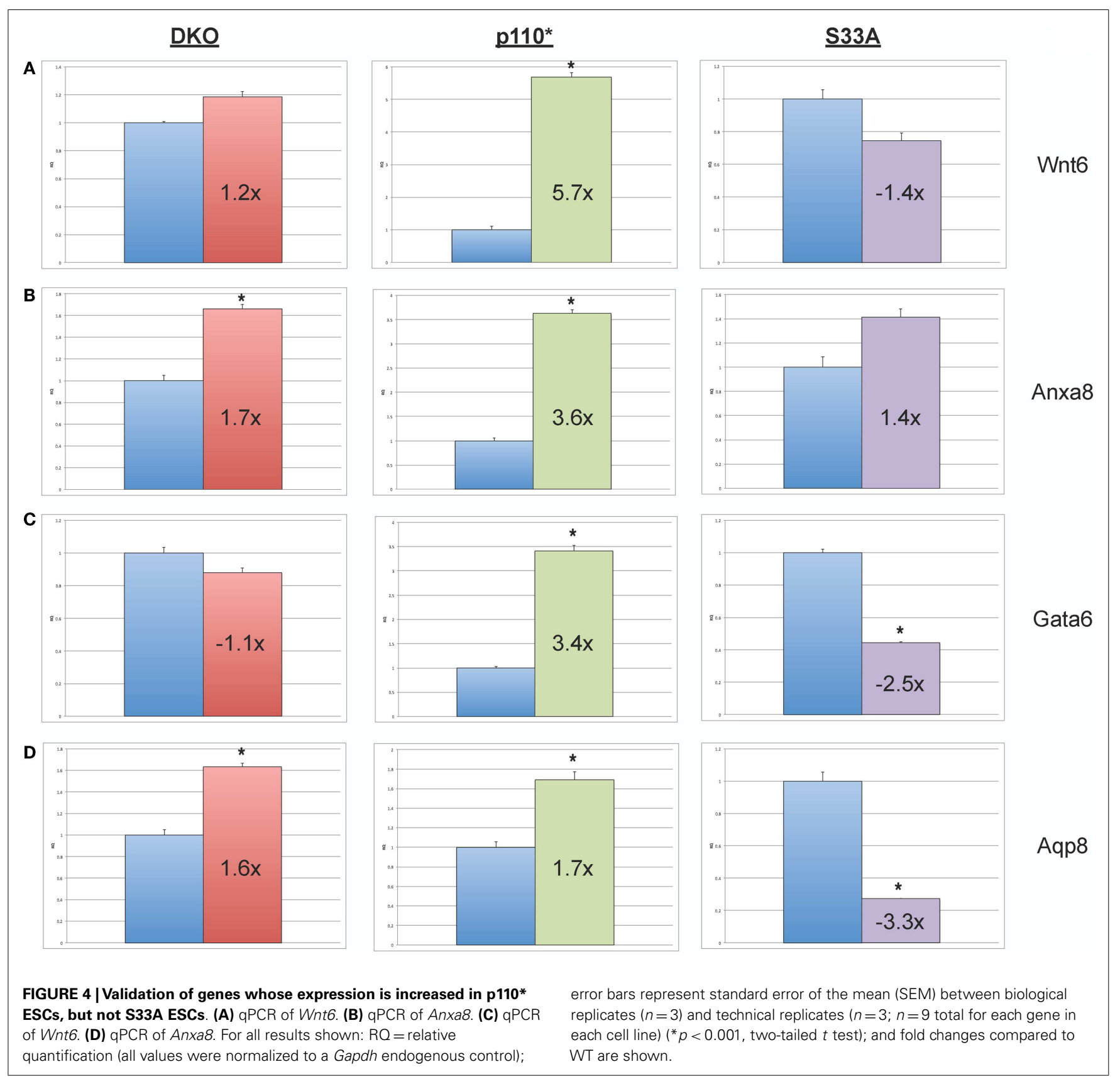

be required to verify whether any of these putative binding sites are bona fide Lef/Tcf binding sites.

Similarly, Bhmt 1 and Bhmt 2 are also contiguous in the mouse genome (13qC3). Neither gene had previously been shown to be regulated by Wnt signaling, but we find both genes have increased expression in Gsk-3 DKO ESCs (14.1- and 13.1-fold, respectively) and even more potently expressed in $\beta$-catenin S33A ESCs (up 70.1- and 56.5-fold, respectively). Bhmt1 and Bhmt2 both encode for betaine-homocysteine $S$-methyltransferase enzymes, participating in the regulation of homocysteine levels (53). Interestingly, variations in $B H M T 1$ and $B H M T 2$ have also been implicated in the development of cleft palate in certain human populations (54-56).

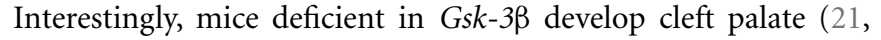
$24,25)$. It is unclear whether this phenotype is a consequence of altered expression of Bhmt1 or Bhmt2, but based on the data presented here, there may be a functional connection between these observations.

While not as dramatic as the changes seen for putative Wnt target genes, we found several genes whose expression was insensitive to constitutively active Wnt signaling, but were increased or decreased in expression in a Gsk-3/PI3K-dependent manner. It has been demonstrated in animal models of various cancers that the transcription factor ZEB1 activates PI3K signaling (5759 ) and this results in increased expression of Gata6 (60). Our 


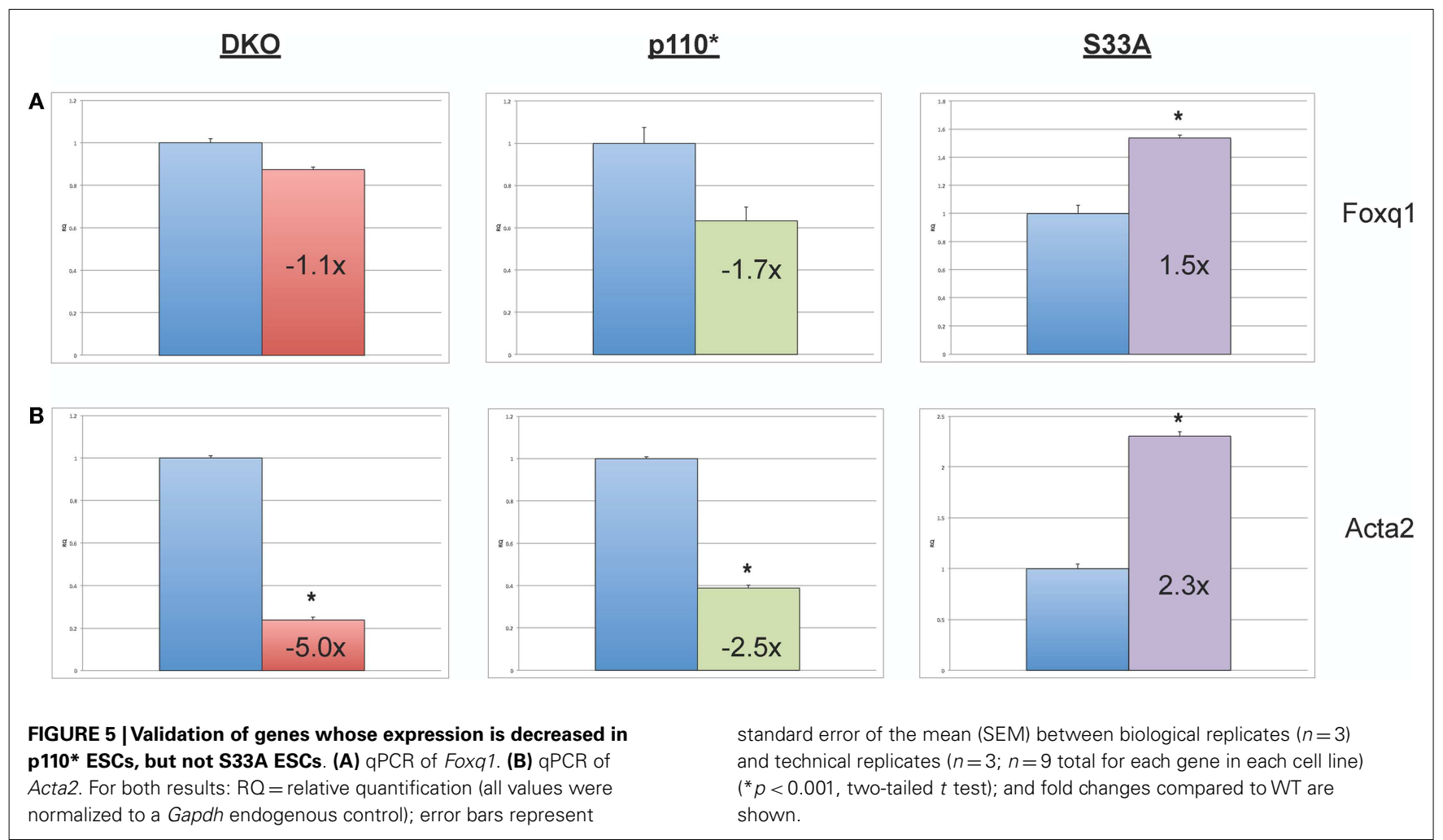

data from mouse ESCs stably expressing a constitutively active form of the p110 subunit of PI $3 \mathrm{~K}$ corroborates these findings and show that the PI3K-mediated increase in Gata6 expression is not limited to cancer cell populations. Wnt6, Anxa8, and Aqp8 also showed increased expression in p110* ESCs, while all but Anxa8 had reduced expression in S33A ESCs. While PI3K signaling has been shown to regulate the subcellular localization of Aqp8 in hepatocytes (61), this is the first report that activation of PI3K signaling has an effect on Aqp8 transcription. Anxa 8 expression has been shown to be down-regulated by epidermal growth factor (EGF)-stimulated PI3K signaling in a model of metastatic cholangiocarcinoma (62), while we find that Anxa8 expression is increased 3.6-fold in p110* ESCs, likely reflecting cell-type differences in Anxa8 gene regulation. It should be mentioned that we selected Gata6, Wnt6, Anxa8, and Aqp8 for validation by qPCR because these genes showed increased expression in both Gsk-3 DKO and $110^{*}$ ESCs by microarray; however, the qPCR results showed only modest changes in gene expression in the Gsk-3 DKO ESCs, highlighting the importance of not relying on microarray gene expression data alone.

Finally, we also found that the expression of Foxq1 and Acta2 were decreased in p110* ESCs. Acta2 showed the most robust changes in expression (down fivefold in Gsk-3 DKO ESCs, down 2.5 -fold in p110* ESCs, and up 2.3-fold in S33A ESCs). We did find that Foxq1 expression is modestly decreased in p110* ESCs and increased 1.5-fold in S33A ESCs. Foxq1 has recently been shown to be one of the most highly expressed genes in human colorectal cancer and has been shown to be a direct target of Wnt signaling, which is often constitutively activated in colorectal cancer cells (63). Based on these data, we expected to find increased Foxq1 expression in both Gsk-3 DKO and S33A ESCs, but we did not observe these expected increases. It will be interesting to determine the mechanism responsible for the lack of response by Foxq1 to constitutively activated Wnt signaling in the context of mouse ESCs.

Taken together, our data shows that, while many genes have their expression changed in Gsk-3 DKO ESCs, only a fraction of the changes we observe are due to activated Wnt signaling or activated PI3K signaling, meaning that there are likely several other signaling pathways whose activation affects Gsk-3-dependent gene expression. Thus, our data provide a framework for future analyses to make these connections. Furthermore, while these studies were performed in mouse ESCs, we believe that the findings from this study can be used in future studies examining the role of insulin or Wnt signaling pathways in other biological settings, such as the study of insulin resistance in the diabetic brain.

\section{ACKNOWLEDGMENTS}

This work was supported by NIH R01AG031883 and funds from the University of Colorado Denver.

\section{SUPPLEMENTARY MATERIAL}

The Supplementary Material for this article can be found online at http://www.frontiersin.org/Journal/10.3389/fendo.2014.00133/ abstract

Datasheet 1 | Microarray gene expression data for Gsk-3 DKO ESCs, $\beta$-catenin S33A ESCs and p110* ESCs. Tabs at the bottom of the spreadsheet contain the microarray data, either as All Data, or sorted by fold changes in expression as seen in Gsk-3 DKO, p110*, or $\beta$-catenin S33A ESCs. Within each tab is found the Agilent probe ID, Gene symbol, Gene name, NCBI accession 
number, Entrez Gene ID, fold-changes (FC) compared to WT ESCs, false-discovery rates (FDR) compared to WT ESCs, and adjusted P-values (AdjP) compared to WT ESCs. The normalized microarray values for each individual sample, as well as the averages, are also shown

\section{REFERENCES}

1. Embi N, Rylatt DB, Cohen P. Glycogen synthase kinase-3 from rabbit skeletal muscle. Separation from cyclic-AMP-dependent protein kinase and phosphorylase kinase. Eur J Biochem (1980) 107:519-27. doi:10.1111/j.1432-1033.1980. tb06059.x

2. Woodgett JR. Molecular cloning and expression of glycogen synthase kinase3/factor A. EMBO J (1990) 9:2431-8.

3. Buescher JL, Phiel CJ. A noncatalytic domain of glycogen synthase kinase-3 (GSK-3) is essential for activity. J Biol Chem (2010) 285:7957-63. doi:10.1074/ jbc.M109.091603

4. Grimes CA, Jope RS. The multifaceted roles of glycogen synthase kinase 3beta in cellular signaling. Prog Neurobiol (2001) 65:391-426. doi:10.1016/S03010082(01)00024-7

5. Frame S, Cohen P. GSK3 takes centre stage more than 20 years after its discovery. Biochem J (2001) 359:1-16. doi:10.1042/0264-6021:3590001

6. Doble BW, Woodgett JR. GSK-3: tricks of the trade for a multi-tasking kinase. J Cell Sci (2003) 116:1175-86. doi:10.1242/jcs.00384

7. Cross D, Alessi D, Cohen P, Andjelkovich M, Hemmings B. Inhibition of glycogen synthase kinase-3 by insulin mediated by protein kinase B. Nature (1995) 378:785-9. doi:10.1038/378785a0

8. Klippel A, Reinhard C, Kavanaugh WM, Apell G, Escobedo MA, Williams LT Membrane localization of phosphatidylinositol 3-kinase is sufficient to activate multiple signal-transducing kinase pathways. Mol Cell Biol (1996) 16: 4117-27.

9. Popkie AP, Zeidner LC, Albrecht AM, D'Ippolito A, Eckardt S, Newsom DE, et al. Phosphatidylinositol 3-kinase (PI3K) signaling via glycogen synthase kinase3 (Gsk-3) regulates DNA methylation of imprinted loci. J Biol Chem (2010) 285:41337-47. doi:10.1074/jbc.M110.170704

10. Takahashi K, Mitsui K, Yamanaka S. Role of ERas in promoting tumourlike properties in mouse embryonic stem cells. Nature (2003) 423:541-5. doi:10.1038/nature 01646

11. Clevers H, Nusse R. Wnt/beta-catenin signaling and disease. Cell (2012) 149:1192-205. doi:10.1016/j.cell.2012.05.012

12. Rubinfeld B, Albert I, Porfiri E, Fiol C, Munemitsu S, Polakis P. Binding of GSK3beta to the APC-beta-catenin complex and regulation of complex assembly. Science (1996) 272:1023-6. doi:10.1126/science.272.5264.1023

13. Yost C, Torres M, Miller J, Huang E, Kimelman D, Moon R. The axis-inducing activity, stability, and subcellular distribution of b-catenin is regulated in Xenopus embryos by glycogen synthase kinase 3. Genes Dev (1996) 10:1443-54. doi:10.1101/gad.10.12.1443

14. Aberle H, Bauer A, Stappert J, Kispert A, Kemler R. beta-catenin is a target for the ubiquitin-proteasome pathway. EMBO J (1997) 16:3797-804. doi:10.1093/emboj/16.13.3797

15. Zeng X, Tamai K, Doble B, Li S, Huang H, Habas R, et al. mechanism for Wnt co-receptor phosphorylation and activation. Nature (2005) 438:873-7. doi:10.1038/nature 04185

16. Wu G, Huang H, Garcia Abreu J, He X. Inhibition of GSK3 phosphorylation of beta-catenin via phosphorylated PPPSPXS motifs of Wnt coreceptor LRP6. PLoS One (2009) 4:e4926. doi:10.1371/journal.pone.0004926

17. Taelman VF, Dobrowolski R, Plouhinec JL, Fuentealba LC, Vorwald PP, Gumper $\mathrm{I}$, et al. Wnt signaling requires sequestration of glycogen synthase kinase 3 inside multivesicular endosomes. Cell (2010) 143:1136-48. doi:10.1016/j.cell.2010.11. 034

18. Korinek V, Barker N, Morin PJ, van Wichen D, de Weger R, Kinzler KW, et al. Constitutive transcriptional activation by a beta-catenin-Tcf complex in APC/- colon carcinoma. Science (1997) 275:1784-7. doi:10.1126/science.275.5307. 1784

19. Morin PJ, Sparks AB, Korinek V, Barker N, Clevers H, Vogelstein B, et al. Activation of beta-catenin-Tcf signaling in colon cancer by mutations in beta-catenin or APC. Science (1997) 275:1787-90. doi:10.1126/science.275.5307.1787

20. Hoeflich KP, Luo J, Rubie EA, Tsao MS, Jin O, Woodgett JR. Requirement for glycogen synthase kinase-3beta in cell survival and NF- kappaB activation [see comments]. Nature (2000) 406:86-90. doi:10.1038/35017574
21. Liu KJ, Arron JR, Stankunas K, Crabtree GR, Longaker MT. Chemical rescue of cleft palate and midline defects in conditional GSK-3beta mice. Nature (2007) 446:79-82. doi:10.1038/nature05557

22. MacAulay K, Doble BW, Patel S, Hansotia T, Sinclair EM, Drucker DJ, et al. Glycogen synthase kinase 3alpha-specific regulation of murine hepatic glycogen metabolism. Cell Metab (2007) 6:329-37. doi:10.1016/j.cmet.2007.08. 013

23. Doble BW, Patel S, Wood GA, Kockeritz LK, Woodgett JR. Functional redundancy of GSK-3alpha and GSK-3beta in Wnt/beta-catenin signaling shown by using an allelic series of embryonic stem cell lines. Dev Cell (2007) 12:957-71. doi:10.1016/j.devcel.2007.04.001

24. He F, Popkie AP, Xiong W, Li L, Wang Y, Phiel CJ, et al. Gsk3beta is required in the epithelium for palatal elevation in mice. Dev Dyn (2010) 239:3235-46. doi:10.1002/dvdy.22466

25. Barrell WB, Szabo-Rogers HL, Liu KJ. Novel reporter alleles of GSK-3alpha and GSK-3beta. PLoS One (2012) 7:e50422. doi:10.1371/journal.pone.0050422

26. Kim WY, Wang X, Wu Y, Doble BW, Patel S, Woodgett JR, et al. GSK-3 is a master regulator of neural progenitor homeostasis. Nat Neurosci (2009) 12:1390-97. doi: $10.1038 / \mathrm{nn} .2408$

27. Ding VW, Chen RH, McCormick F. Differential regulation of glycogen synthase kinase 3beta by insulin and Wnt signaling. J Biol Chem (2000) 275:32475-81. doi:10.1074/jbc.M005342200

28. McManus EJ, Sakamoto K, Armit LJ, Ronaldson L, Shpiro N, Marquez R, et al. Role that phosphorylation of GSK3 plays in insulin and Wnt signalling defined by knockin analysis. EMBOJ (2005) 24:1571-83. doi:10.1038/sj.emboj.7600633

29. Ng SS, Mahmoudi T, Danenberg E, Bejaoui I, de Lau W, Korswagen HC, et al. Phosphatidylinositol 3-kinase signaling does not activate the wnt cascade. J Biol Chem (2009) 284:35308-13. doi:10.1074/jbc.M109.078261

30. Klein PS, Melton DA. A molecular mechanism for the effect of lithium on development. Proc Natl Acad Sci USA (1996) 93:8455-9. doi:10.1073/pnas.93.16.8455

31. Stambolic V, Ruel L, Woodgett J. Lithium inhibits glycogen synthase kinase-3 activity and mimics wingless signalling in intact cells. Curr Biol (1996) 6:1664-8. doi:10.1016/S0960-9822(02)70790-2

32. Phiel CJ, Klein PS. Molecular Targets of Lithium Action. Annu Rev Pharmacol Toxicol (2001) 41:789-813. doi:10.1146/annurev.pharmtox.41.1.789

33. Ryder J, Su Y, Liu F, Li B, Zhou Y, Ni B. Divergent roles of GSK3 and CDK5 in APP processing. Biochem Biophys Res Commun (2003) 312:922-9. doi:10.1016/j.bbrc.2003.11.014

34. Su Y, Ryder J, Li B, Wu X, Fox N, Solenberg P, et al. Lithium, a common drug for bipolar disorder treatment, regulates amyloid-beta precursor protein processing. Biochemistry (2004) 43:6899-908. doi:10.1021/bi035627j

35. Phiel CJ, Wilson CA, Lee VM, Klein PS. GSK-3a regulates production of Alzheimer's disease amyloid-b peptides. Nature (2003) 423:435-9. doi:10.1038/ nature 01640

36. Noble W, Planel E, Zehr C, Olm V, Meyerson J, Suleman F, et al. Inhibition of glycogen synthase kinase-3 by lithium correlates with reduced tauopathy and degeneration in vivo. Proc Natl Acad Sci USA (2005) 102:6990-5. doi:10.1073/pnas.0500466102

37. Sereno L, Coma M, Rodriguez M, Sanchez-Ferrer P, Sanchez MB, Gich I, et al. A novel GSK-3beta inhibitor reduces Alzheimer's pathology and rescues neuronal loss in vivo. Neurobiol Dis (2009) 35:359-67. doi:10.1016/j.nbd.2009.05.025

38. Hurtado DE, Molina-Porcel L, Carroll JC, Macdonald C, Aboagye AK, Trojanowski JQ, et al. Selectively silencing GSK-3 isoforms reduces plaques and tangles in mouse models of Alzheimer's disease. J Neurosci (2012) 32:7392-402. doi:10.1523/JNEUROSCI.0889-12.2012

39. Kelly KF, Ng DY, Jayakumaran G, Wood GA, Koide H, Doble BW. beta-catenin enhances Oct-4 activity and reinforces pluripotency through a TCF-independent mechanism. Cell Stem Cell (2011) 8:214-27. doi:10.1016/j.stem.2010.12.010

40. Schmittgen TD, Livak KJ. Analyzing real-time PCR data by the comparative C(T) method. Nat Protoc (2008) 3:1101-8. doi:10.1038/nprot.2008.73

41. Gentleman RC, Carey VJ, Bates DM, Bolstad B, Dettling M, Dudoit S, et al. Bioconductor: open software development for computational biology and bioinformatics. Genome Biol (2004) 5:R80. doi:10.1186/gb-2004-5-10-r80

42. Bolstad BM, Irizarry RA, Astrand M, Speed TP. A comparison of normalization methods for high density oligonucleotide array data based on variance and bias. Bioinformatics (2003) 19:185-93. doi:10.1093/bioinformatics/19.2.185

43. Tusher VG, Tibshirani R, Chu G. Significance analysis of microarrays applied to the ionizing radiation response. Proc Natl Acad Sci USA (2001) 98:5116-21. doi:10.1073/pnas.091062498 
44. Storey JD, Tibshirani R. Statistical significance for genomewide studies. Proc Natl Acad Sci USA (2003) 100:9440-5. doi:10.1073/pnas.1530509100

45. Benjamini Y, Hochberg Y. Controlling the false discovery rate: a practical and powerful approach to multiple testing. J R Stat Soc B (1995) 57:289-300.

46. Chen J, Bardes EE, Aronow BJ, Jegga AG. ToppGene Suite for gene list enrichment analysis and candidate gene prioritization. Nucleic Acids Res (2009) 37:W305-11. doi:10.1093/nar/gkp427

47. MacDonald BT, Tamai K, He X. Wnt/beta-catenin signaling: components, mechanisms, and diseases. Dev Cell (2009) 17:9-26. doi:10.1016/j.devcel.2009.06.016

48. Jho EH, Zhang T, Domon C, Joo CK, Freund JN, Costantini F. Wnt/betacatenin/Tcf signaling induces the transcription of Axin2, a negative regulator of the signaling pathway. Mol Cell Biol (2002) 22:1172-83. doi:10.1128/MCB. 22.4.1172-1183.2002

49. Zhao T, Gan Q, Stokes A, Lassiter RN, Wang Y, Chan J, et al. beta-catenin regulates Pax3 and Cdx2 for caudal neural tube closure and elongation. Development (2014) 141:148-57. doi:10.1242/dev.101550

50. Soichot M, Hennart B, Al Saabi A, Leloire A, Froguel P, Levy-Marchal C, et al. Identification of a variable number of tandem repeats polymorphism and characterization of LEF-1 response elements in the promoter of the IDO1 gene. PLoS One (2011) 6:e25470. doi:10.1371/journal.pone.0025470

51. Messeguer X, Escudero R, Farre D, Nunez O, Martinez J, Alba MM. PROMO: detection of known transcription regulatory elements using species-tailored searches. Bioinformatics (2002) 18:333-4. doi:10.1093/bioinformatics/18.2.333

52. Farre D, Roset R, Huerta M, Adsuara JE, Rosello L, Alba MM, et al. Identification of patterns in biological sequences at the ALGGEN server: PROMO and MALGEN. Nucleic Acids Res (2003) 31:3651-3. doi:10.1093/nar/gkg605

53. Pajares MA, Perez-Sala D. Betaine homocysteine S-methyltransferase: just a regulator of homocysteine metabolism? Cell Mol Life Sci (2006) 63:2792-803. doi:10.1007/s00018-006-6249-6

54. Hu Y, Chen E, Mu Y, Li J, Chen R. BHMT gene polymorphisms as risk factors for cleft lip and cleft palate in a Chinese population. Biomed Environ Sci (2011) 24:89-93. doi:10.3967/0895-3988.2011.02.001

55. Martinelli M, Masiero E, Carinci F, Morselli PG, Pezzetti F, Scapoli L. New evidence for the role of cystathionine beta-synthase in non-syndromic cleft lip with or without cleft palate. Eur J Oral Sci (2011) 119:193-7. doi:10.1111/j. 1600-0722.2011.00824.x

56. Mostowska A, Hozyasz KK, Biedziak B, Misiak J, Jagodzinski PP. Polymorphisms located in the region containing BHMT and BHMT2 genes as maternal protective factors for orofacial clefts. Eur J Oral Sci (2010) 118:325-32. doi:10.1111/j.1600-0722.2010.00757.x

57. Gupta S, Ramjaun AR, Haiko P, Wang Y, Warne PH, Nicke B, et al. Binding of ras to phosphoinositide 3-kinase p110alpha is required for ras-driven tumorigenesis in mice. Cell (2007) 129:957-68. doi:10.1016/j.cell.2007.03.051
58. Yang Y, Iwanaga K, Raso MG, Wislez M, Hanna AE, Wieder ED, et al. Phosphatidylinositol 3-kinase mediates bronchioalveolar stem cell expansion in mouse models of oncogenic K-ras-induced lung cancer. PLoS One (2008) 3:e2220. doi:10.1371/journal.pone.0002220

59. Dubrovska A, Kim S, Salamone RJ, Walker JR, Maira SM, Garcia-Echeverria C, et al. The role of PTEN/Akt/PI3K signaling in the maintenance and viability of prostate cancer stem-like cell populations. Proc Natl Acad Sci USA (2009) 106:268-73. doi:10.1073/pnas.0810956106

60. Yang Y, Ahn YH, Chen Y, Tan X, Guo L, Gibbons DL, et al. ZEB1 sensitizes lung adenocarcinoma to metastasis suppression by $\mathrm{PI} 3 \mathrm{~K}$ antagonism. J Clin Invest (2014) 124:2696-708. doi:10.1172/JCI72171

61. Gradilone SA, Carreras FI, Lehmann GL, Marinelli RA. Phosphoinositide 3-kinase is involved in the glucagon-induced translocation of aquaporin-8 to hepatocyte plasma membrane. Biol Cell (2005) 97:831-6. doi:10.1042/ BC20040115

62. Lee MJ,Yu GR, Yoo HJ, Kim JH, Yoon BI, Choi YK, et al. ANXA8 down-regulation by EGF-FOXO4 signaling is involved in cell scattering and tumor metastasis of cholangiocarcinoma. Gastroenterology (2009) 137:1138-50. doi:10.1053/ j.gastro.2009.04.015

63. Christensen J, Bentz S, Sengstag T, Shastri VP, Anderle P. FOXQ1, a novel target of the Wnt pathway and a new marker for activation of Wnt signaling in solid tumors. PLoS One (2013) 8:e60051. doi:10.1371/journal.pone.0060051

Conflict of Interest Statement: The authors declare that the research was conducted in the absence of any commercial or financial relationships that could be construed as a potential conflict of interest.

Received: 09 June 2014; paper pending published: 27 June 2014; accepted: 28 July 2014; published online: 13 August 2014.

Citation: Bartman CM, Egelston J, Kattula S, Zeidner LC, D'Ippolito A, Doble $B W$ and Phiel CJ (2014) Gene expression profiling in mouse embryonic stem cells reveals glycogen synthase kinase-3-dependent targets of phosphatidylinositol 3-kinase and Wnt/ $\beta$-catenin signaling pathways. Front. Endocrinol. 5:133. doi: $10.3389 /$ fendo.2014.00133

This article was submitted to Diabetes, a section of the journal Frontiers in Endocrinology.

Copyright (c) 2014 Bartman, Egelston, Kattula, Zeidner, D'Ippolito, Doble and Phiel. This is an open-access article distributed under the terms of the Creative Commons Attribution License (CC BY). The use, distribution or reproduction in other forums is permitted, provided the original author(s) or licensor are credited and that the original publication in this journal is cited, in accordance with accepted academic practice. No use, distribution or reproduction is permitted which does not comply with these terms. 